\title{
Impact of exercise training on sarcopenia associated with non-alcoholic fatty liver disease (NAFLD) in humans: A systematic review and meta-analysis
}

\author{
Andrea Gonzalez ${ }^{1,2,3}$, Mayalen Valero-Breton ${ }^{1,2,3}$, Camila Huerta-Salgado ${ }^{1,2,3}$, Oscar Achiardi ${ }^{6}$, Felipe Simon ${ }^{2,4,5}$, and \\ Claudio Cabello-Verrugio ${ }^{1,2,3^{*}}$
}

\footnotetext{
${ }^{1}$ Laboratory of Muscle Pathology, Fragility and Aging, Department of Biological Sciences, Faculty of Life Sciences. Universidad Andres Bello. Santiago, 8370146, Chile.

${ }^{2}$ Millennium Institute on Immunology and Immunotherapy. Santiago, 8370146, Chile.

${ }^{3}$ Center for the Development of Nanoscience and Nanotechnology (CEDENNA), Universidad de Santiago de Chile. Santiago, 8350709, Chile.

${ }^{4}$ Millennium Nucleus of Ion Channels-Associated Diseases (MiNICAD), Universidad de Chile, Santiago 8370146, Chile

${ }^{5}$ Laboratory of Integrative Physiopathology, Department of Biological Sciences, Faculty of Life Sciences. Universidad Andres Bello. Santiago, 8370146, Chile

${ }^{6}$ Escuela de Kinesiología, Facultad de Ciencias, Pontificia Universidad Católica de Valparaíso. Valparaíso, 2340025, Chile.
}

* Corresponding author: Claudio Cabello-Verrugio. Laboratory of Muscle Pathology, Fragility, and Aging. Department of Biological Sciences. Faculty of Life Sciences. Universidad Andres Bello. Santiago, 8370146, Chile.

Keywords: sarcopenia, non-alcoholic fatty liver disease (NAFLD), exercise training, muscle mass, muscle strength, physical performance

\begin{abstract}
Objective: To conduct a systematic review and meta-analyses to assess the efficacy of physical exercise on strength, muscle mass and physical function in adult patients with non-alcoholic fatty liver disease (NAFLD).

Design: We conducted a systematic review and meta-analysis of seven studies to investigate the effect of exercise training interventions in muscle strength, muscle mass and physical performance.

Data sources: We identified relevant randomised controlled trials (RCT) in electronic databases (PubMed, CINAHL and Scopus).

Eligibility criteria: We selected seven RCTs from 66 screened studies. The inclusion criteria were peerreviewed and English writing articles that included adult patients with liver disease of non-alcoholic origin, applied resistance training, endurance training or both, and assayed at least one variable of sarcopenia.

Results: Physical performance criterion improved in the exercise groups (mean differences [MD] 8.26 $\mathrm{mL} / \mathrm{Kg} * \min$ [ $95 \% \mathrm{Cl} 5.27$ to $11.24 \mathrm{~mL} / \mathrm{Kg} * \min$ ], $\mathrm{p}<0.0001$ ) versus the control groups; muscle mass, determined as lean body mass (LBM), showed no evidence of the beneficial effects of exercise versus the control groups (MD $1.01 \mathrm{Kg}$ [95\% $\mathrm{Cl}-1.78$ to $3.80 \mathrm{Kg}$ ], $\mathrm{p}=0.48$ ); we did not include muscle strength, as none of the selected studies evaluated it.

Summary/conclusion: Exercise training is a useful intervention strategy to treat sarcopenia in patients with NAFLD; it increases their physical performance in the form of aerobic capacity but does not affect LBM. Future research should include muscle strength assessments and resistance training to evaluate the effects of exercise training on sarcopenia in NAFLD patients.

PROSPERO reference number CRD42020191471
\end{abstract}




\section{INTRODUCTION}

Non-alcoholic fatty liver disease (NAFLD) is the most common type of chronic liver disease (CLD). It includes a broad spectrum of disorders ranging from accumulating lipids in the liver (steatosis) to the progressive inflammation denoted as non-alcoholic steatohepatitis (NASH) to advanced stages of damage such as fibrosis and cirrhosis. ${ }^{12}$

Sarcopenia is one of the most common complications associated with NAFLD, with a prevalence of $30-70 \% .^{3}$ According to the European Working Group on Sarcopenia in Older People (EWGSOP), sarcopenia diagnosis is based on three criteria: i) low muscle strength (the primary indicator of sarcopenia), ii) small muscle quantity or quality and iii) low physical performance. ${ }^{4}$ Sarcopenia negatively affects NAFLD progression; ${ }^{5}$ its severity is more pronounced in the advanced stages of NAFLD, considered as an independent predictor of pre- and post-liver transplant complications and mortality. ${ }^{67}$ Therefore, therapeutic interventions to prevent, revert or improve sarcopenia associated with NAFLD are essential.

Since sarcopenia involves a decline in muscle mass, strength and physical function, physical exercise is a promising tool for treating sarcopenia. ${ }^{8}$ Due to low-quality evidence, the results of studies that evaluate the effect of exercise training on patients with cirrhosis-associated sarcopenia are highly variable and inconclusive. ${ }^{9}$ Despite evidence that training has a positive effect on liver dysfunction parameters in patients with NAFLD, ${ }^{10} 11$ no studies evaluate the impact of training on sarcopenia parameters in NAFLD patients. In this context, a recent narrative review concluded that there is no consensus on the best type of exercise to improve muscle strength and physical function in NAFLD patients. Thus, more evidence is required to determine exercise specifications (type, intensity, frequency, supervised vs domiciliary). ${ }^{2}$

Considering all the antecedents, we conclude that the effects of exercise training on sarcopenia associated with NAFLD are poorly determined and that there is no consensus on the best type of exercise for these patients. Therefore, our objective was to conduct a systematic review and meta-analyses to assess the efficacy of physical exercise on strength, muscle mass and physical function in adult patients with NAFLD.

\section{METHODOLOGY}

\section{Protocol and Registration}

We conducted our systematic review and meta-analysis following the Preferred Reporting Items for Systematic Reviews and Meta-Analysis (PRISMA) statement. We registered the review protocol in the International Prospective Register of Systematic Reviews (PROSPERO reference number CRD42020191471), available from 3 September 2020.

\section{Search strategy}

We performed a systematic search in PubMed, CINAHL and Scopus from March-May 2020. We included articles published from January 2000-March 2020 containing the critical concepts of sarcopenia, fatty liver disease, exercise and related words. The essential concepts of the search were different for each database. For example, for PubMed, we used the keywords ('atrophic muscular disorders' OR 'muscle atrophy' OR 'muscle degeneration' OR 'muscle fiber atrophy' OR 'muscle fiber degeneration' OR 'muscle wasting' OR 'muscular wasting' OR 'muscular atrophy' OR 'muscular atrophies' OR 'muscular degeneration' OR 'sarcopenia') AND ('non-alcoholic steatosis' OR 'non-alcoholic fatty liver disease' OR 'fatty liver' OR 'hepatic fat' OR 'liver fibrosis' OR 'liver disease' OR 'fatty liver disease' OR 'obesity') AND ('exercise' OR 'physical activity' OR 'exercise intervention' OR 'training') (see Supplementary Material 1). We filtered the results for clinical randomised controlled trials (RCTs). Then, AG reviewed all titles and abstracts from the articles selected and only downloaded those matching the inclusion criteria. 
medRxiv preprint doi: https://doi.org/10.1101/2020.09.05.20189100; this version posted September 11, 2020. The copyright holder for this preprint (which was not certified by peer review) is the author/funder, who has granted medRxiv a license to display the preprint in perpetuity.

It is made available under a CC-BY-NC-ND 4.0 International license .

\section{Inclusion/exclusion criteria}

The inclusion criteria are based on the study population, intervention, comparison question (control) and outcomes (PICO). ${ }^{12}$

- Investigate adults (18 or older) with liver disease of non-alcoholic origin (specifically, NAFLD or NASH) as confirmed by at least one of these parameters: hepatic biopsy, ultrasonography, computed tomography (CT), nuclear magnetic resonance spectroscopy (MRS), serum bile acids, gamma-glutamyl transpeptidase (GGT), aminotransferases, the ratio of aspartate transaminase (AST)/alanine transaminase (ALT) or bilirubin, alkaline phosphatase, dyslipidaemia) ${ }^{13-17}$ (Population).

- Apply resistance training, endurance training or both. Combined exercise is defined as interventions that simultaneously used resistance and endurance training (Intervention).

- Apply exercise alone or in combination with other interventions (educational, nutritional, etc.); must have a control group and a group with exercise only (to determine its independent effect) (Control). Control groups cannot have any intervention that could influence study outcome measures (e.g. nutritional or physical intervention). Moreover, participants of the exercise group must perform three or more sessions of training per week for at least four weeks to determine the chronic effect of exercise on the variables. Studies must conduct preand post-intervention assessments to identify changes in the variables.

- Assay at least one variable included in the consensus definition of sarcopenia: ${ }^{2} 18$ muscle strength, defined as the force generated through muscular contraction against an external load; ${ }^{19}$ muscle mass, defined as the part of total body mass composed of skeletal muscle tissue; ${ }^{20}$ and physical performance, defined as an objectively measured whole body function related with mobility. ${ }^{21}$ Thus, the studies must evaluate any of three criteria, as it was described in Table 1 (Outcome).

- Be a peer-reviewed study

- Be written in English

Exclusion criteria:

- Bibliographic reviews, not controlled or randomised clinical trials, animal models

- The participants had comorbidities such as type II diabetes mellitus, viral hepatitis, cardiovascular disease, lung disease or neurological disease.

- Interventions that would interfere with identifying any exercise-mediated effects

Two researchers ( $\mathrm{CH}-\mathrm{S}$ and $\mathrm{MV}-\mathrm{B}$ ) independently reviewed the full-text version of the reports. They resolved discrepancies/disagreements by consensus - when this was not possible, two independent researchers acted as referees (AG and CC-V).

\section{Table 1. Assessment criteria for the diagnosis of sarcopenia in NAFLD}

\begin{tabular}{|l|l|l|}
\multicolumn{1}{|c|}{$\begin{array}{c}\text { Body composition } \\
\text { (muscular mass) }\end{array}$} & \multicolumn{1}{|c|}{ Muscular Strength } & \multicolumn{1}{c|}{ Physical Performance } \\
\hline $\begin{array}{l}\text { Extremity circumferences (Thigh, } \\
\text { Arm) }\end{array}$ & Handgrip strength & 6-MWT (6-minute walk test) \\
\hline Thigh US (ultrasound) & Knee flexion/extension & 2-MST (2-minute step test) \\
\hline
\end{tabular}


medRxiv preprint doi: https://doi.org/10.1101/2020.09.05.20189100; this version posted September 11, 2020. The copyright holder for this preprint (which was not certified by peer review) is the author/funder, who has granted medRxiv a license to display the preprint in perpetuity.

It is made available under a CC-BY-NC-ND 4.0 International license .

\begin{tabular}{|c|c|c|}
\hline $\begin{array}{l}\text { BIA (bioelectrical impedance } \\
\text { analysis) }\end{array}$ & Dynamometer & $\begin{array}{l}\text { CPET (cardiopulmonary } \\
\text { exercise testing) }\end{array}$ \\
\hline $\begin{array}{l}\text { CSA (the cross-sectional area from } \\
\text { magnetic resonance imaging) }\end{array}$ & 1 maximum repetition (1RM) & $\begin{array}{l}\text { SPPB (Short physical } \\
\text { performance Battery) }\end{array}$ \\
\hline $\begin{array}{l}\text { DXA (dual-energy x-ray } \\
\text { absorptiometry) }\end{array}$ & $\begin{array}{l}10 \text { maximum repetition } \\
(10 \mathrm{RM})\end{array}$ & Usual gait speed \\
\hline Anthropometry & Isokinetic evaluation & Chair stands \\
\hline \multirow[t]{2}{*}{ MAMA (middle-arm muscle area) } & \multirow[t]{2}{*}{$\begin{array}{l}\text { Peak expiratory flow (specific } \\
\text { to respiratory) }\end{array}$} & Timed get-up-and-go test \\
\hline & & Stair climb power test \\
\hline
\end{tabular}

Table 1. Measurement of sarcopenia criteria in NAFLD patients: muscle strength, muscle mass and physical performance in clinical practice and research.

\section{Data extraction}

We collected the data from seven final papers $(n=7)$, which we tabulated and ordered into a Microsoft Excel 2016 database. One researcher extracted the data, and two different researchers reviewed it to ensure data processing accuracy (Supplementary Material 2). The data included the following parameters: first author's surname; publication year; number of participants by group; participants' sex and age; method used to diagnose NAFLD and its severity; type, intensity, frequency and duration of the exercise intervention; sarcopenia criteria assessment methods. When studies presented intention-to-treat (ITT) values, we only extracted ITT data. Data extraction and synthesis for the outcome of interest are shown in the data synthesis section.

\section{TESTEX rating scale application}

We assessed study quality using the TESTEX rating scale (Table 2). ${ }^{22}$ It includes criteria to assess methodological quality as well as ranking the whole article. The scale comprises 12 assessment criteria for a maximum score of 15 points. Higher ratings reflect better study quality and reporting ${ }^{22}$.

TESTEX does not yet provide a validated cutoff score, ${ }^{22}$ so we categorised the studies depending on the median score. ${ }^{1023}$ We classified studies above the median score as 'high-quality' and those below as 'low-quality'. AG and OA performed TESTEX scoring separately. When different evaluations occurred, they discussed discrepancies until reaching a consensus score.

\begin{tabular}{|c|c|c|c|c|c|c|c|c|c|c|c|c|c|}
\hline Study \Score & 1 & 2 & 3 & 4 & 5 & 6 & 7 & 8 & 9 & 10 & 11 & 12 & TOTAL \\
\hline 2012 Sullivan & 1 & 1 & 1 & 1 & 0 & 2 & 0 & 2 & 1 & 0 & 1 & 1 & 11 \\
\hline 2013 Pugh & 1 & 0 & 1 & 1 & 0 & 3 & 0 & 2 & 1 & 0 & 1 & 1 & 11 \\
\hline 2014 Pugh & 1 & 1 & 1 & 1 & 0 & 1 & 0 & 2 & 1 & 0 & 1 & 1 & 10 \\
\hline 2015 Hallsworth & 1 & 1 & 1 & 1 & 0 & 0 & 0 & 2 & 1 & 0 & 1 & 1 & 9 \\
\hline 2016 Shojaee-Moradie & 1 & 1 & 1 & 1 & 0 & 1 & 0 & 2 & 1 & 0 & 1 & 1 & 10 \\
\hline 2017 Cheng & 1 & 1 & 1 & 1 & 1 & 2 & 1 & 2 & 1 & 0 & 1 & 1 & 13 \\
\hline 2017 Hughton & 1 & 1 & 1 & 1 & 0 & 3 & 0 & 2 & 1 & 0 & 1 & 1 & 12 \\
\hline
\end{tabular}


Table 2. Study quality.

Notes. 1) eligibility criteria specified (1 point); 2 ) randomisation defined (1 point); 3) allocation concealment (1 point); 4$)$ groups similar at baseline (1 point); 5 ) assessor blinding in study reporting (1 point); 6 ) outcome measures assessed in $85 \%$ of patients ( 3 points); 7) intention-to-treat analysis (1 point); 8) between-group statistical comparisons reported (2 points); 9 ) point measures and measures of variability for all reported outcome measures (1 point); 10 ) activity monitoring in the control group (1 point); 11 ) relative exercise intensity remained constant (1 point); 12) exercise volume and energy expenditure (1 point).

\section{Data synthesis}

When at least three included studies reported the same outcome, we pooled data to perform a metaanalysis using Review Manager 5.4 (RevMan 5.4) as per the Cochrane Manual for Systematic Reviews of Interventions. ${ }^{24}$ We used a random-effects model for heterogeneity to address study participants' different backgrounds and the variety of exercise interventions (which create differences in training variables such as exercise intensity and duration, session frequency, etc.). Outcome measures also differ among studies depending on the available technology to quantify muscle mass and physical performance. Finally, we expected methodological heterogeneity, as exercise training research uses different designs.

We used inverse variance because we analysed continuous data to compare exercise vs control or conventional care groups using mean and standard deviations from post-intervention values for each outcome. When other forms of variability measures such as standard error or confidence intervals $(\mathrm{Cl})$ appeared, we calculated the standard deviations using RevMan 5.4. ${ }^{24}$

We did not plan to investigate subgroups of patients, as we expected the participants to be homogeneous (see inclusion criteria); however, considering the heterogeneous nature of exercise training protocols, we expected to find different types of exercise training, which are classified as either endurance or resistance. In the case of this variety, we planned to perform a subgroup analysis comparing endurance training vs resistance training, investigating heterogeneity across subgroup results and analysing the variability in effect estimates between types of exercise using an $I^{2}$ statistic.

For each outcome analysed, we present the data as mean differences (MD) with a $95 \% \mathrm{Cl}$. We used standardised mean differences (SMD) to represent effect sizes and facilitate interpretations, ${ }^{25}$ with a value of 0.2 set as small, 0.5 as moderate and 0.8 or higher as a large effect size. ${ }^{26}$ Positive values indicated that it was favourable to the exercise intervention, and vice versa. Zero value suggested that there was no effect on the analysed variable.

\section{Heterogeneity}

We quantified heterogeneity using the $\chi^{2}$ test, where a significant $p$-value was indicative of more considerable heterogeneity. We also applied a complementary inconsistency test $I^{2}$ and presented this with its corresponding $95 \% \mathrm{Cl}$ to assess the degree of heterogeneity. Higher $\mathrm{I}^{2}$ values indicated greater heterogeneity. ${ }^{27}$

\section{Risk of Bias}

We assessed all the included RCTs using the Cochrane collaboration risk of bias tool to search for elements that could over- or under-estimate the intervention's effect. We evaluated the following for each study: selection bias (random sequence and generations, allocation concealment), performance bias (blinding of participants and research staff), detection bias (blinding of outcome evaluation), attrition bias (incomplete outcome data), reporting bias (selective reporting), other sources of bias. ${ }^{24}$ Two independent reviewers ( $A G$ and $O A$ ) performed the assessments; they discussed discrepancies until reaching a consensus.

\section{RESULTS \\ Review studies}


We initially identified 66 articles in PubMed, CINAHL and Scopus. Of these, we excluded one by duplication and 29 after analysing the title and abstract. Among the remaining 36 articles, we excluded 29 due to the combination of exercise with another intervention $(n=15)$, participants under 18 years $(n=2)$, article written in Spanish $(n=1)$, reports not RCTs $(n=5)$, no determination or evaluation of sarcopenia criteria $(n=2)$ and patients were not diagnosed with NAFLD or had comorbidities $(n=4)$. Supplementary Material 3 lists the excluded studies and causes for the exclusion. ${ }^{28-55}$ We included seven articles in the final analysis $^{56-62}$ (Figure 1).

\section{Description of included studies}

A total of 182 participants were included in the analysis. Among them, 98 participated in exercise interventions and 84 in control groups (see Supplementary Material 2). Five studies used a moderateintensity protocol of endurance training. ${ }^{56-59} 62$ Three studies used a combined exercise protocol (endurance and resistance) ${ }^{59-61}$ In all seven studies the exercise interventions were supervised. All the studies had a control group composed of patients with NAFLD following standard or conventional care, which did not include exercise intervention or any other component that could have influenced the outcomes of interest. ${ }^{56-62}$

Exercise intervention duration ranged from 12-16 weeks, with training frequency of 2-5 times per week lasting 20-60 min per session. Endurance training intensity varied from $45-75 \%$ of oxygen consumption peak $\left(\mathrm{VO}_{2 \text { peak }}\right.$ ), 30-60\% of heart rate reserve (HRR) or 16-18/20 (Borg Scale) rating of perceived exertion (RPE). Endurance training varied: cycle ergometer, treadmill, Nordic brisk walking, walking and self-selected gymnasium exercise routines.

Two articles described high-intensity interval training (HIIT) as a form of endurance training. One of these articles used 5 intervals of 2 min, adding $10 \mathrm{~s}$ to each range per week with 3 min of recovery and an RPE (Borg Scale) intensity of 16-17 (very hard)..$^{60}$ The other article used cycling intervals with an RPE (Borg scale) intensity of 16-18. ${ }^{61}$ The remaining five studies carried out continuous training. ${ }^{56-59} 62$

Among the three articles that carried out combined training, one performed a moderate-intensity endurance protocol ( $40 \%-60 \%$ HRR) combined with resistance training; however, the details of the latter training were not described. ${ }^{59}$ Another study used combined training protocol by performing endurance interval training with an intensity based on RPE of 16-18 (very hard) together with resistance training with an RPE intensity of 14-16 (hard). The exercises included hip and knee extensions, horizontal rows, chest presses, vertical rows and knee extensions. ${ }^{61}$ One article required participants to perform an interval on a cycle ergometer, followed by a light-band-resisted upper body exercise (60 s) in the following order: facepull, horizontal push, horizontal pull, $30^{\circ}$ push. However, it was unclear whether this protocol was a combined workout. ${ }^{60}$

\section{Quality assessment analysis}

The TESTEX scale analysis yielded a median score of 11 out of 15 possible points for the studies, ${ }^{10}$ with values closer to 15 of higher quality than values closer to 1 . We evaluated assessment quality by organising the studies as low-quality if their score was less than the median and high-quality if the score was greater than or equal to the median. ${ }^{10}$ Accordingly, six studies were high quality and one article was low-quality (Table 2). The main weaknesses TESTEX revealed were the supervision of physical activity in the control groups ( 0 of 7 articles), intention-to-treat analysis ( 1 of 7 studies) and blinding of the assessor for at least one key outcome (1 of 7 articles).

\section{Risk of bias assessment}

The risk of bias was low in the RCTs included in this systematic review and meta-analyses for every outcome. However, we had some concerns about their analysis of physical performance regarding blinding the assessors. Only one of the studies performed this key methodological feature, indicating a potential 
risk of detection bias for articles that used a cardiopulmonary exercise test (CPET) to assess physical performance, as assessors might influence patients' performance in this type of evaluation. The details about the risk of bias assessment can be found in Supplementary Materials 4 and 5 .

\section{Change in muscle strength}

We excluded the muscle strength criterion for sarcopenia because none of the selected studies evaluated it.

\section{Change in physical performance}

For the physical performance criterion, we performed a meta-analysis with the results obtained postexercise by comparing the control group vs the exercise group. Data on physical performance were present in four studies, ${ }^{56-59}$ all of which determined the direct maximum oxygen consumption $\left(\mathrm{VO}_{2 \max }\right)$ or oxygen consumption peak $\left(\mathrm{VO}_{2 \text { peak }}\right.$ ) based on CPET and expressed in $\mathrm{mL} / \mathrm{Kg}^{*} \mathrm{~min}$. In this regard, we excluded one article because it performed the $2 \mathrm{Km}$ walking test, which does not directly evaluate physical performance. ${ }^{62}$

Three studies showed significant increases in $\mathrm{VO}_{2 \max }$ or $\mathrm{VO}_{2 \text { peak. }} .57-59$ One study showed no change; here, the authors recognised that the results could be explained by a type II statistical error because of the small number of participants. ${ }^{56}$

The pooled analysis of the studies indicated a change in physical performance in favour of exercise (MD $8.26 \mathrm{~mL} / \mathrm{Kg}^{*} \min \left[95 \% \mathrm{Cl} 5.27-11.24 \mathrm{~mL} / \mathrm{Kg}^{*} \mathrm{~min}\right], \mathrm{p}<0.0001$, Figure 2) and a large effect size (SMD $\left.1.10[0.60-1.60], p<0.0001, l^{2}=0 \%\right)$. Studies that analysed physical performance had low heterogeneity $\left(\mathrm{Chi}^{2} 2.43, \mathrm{p}=0.49 ; \mathrm{I}^{2} 0 \% ; \mathrm{Tau}^{2}=0.00\right)$.

\section{Change in muscle mass}

Four studies determined muscle mass as LBM. ${ }^{560-62}$ They evaluated LBM by different methods, including dual-energy X-ray absorptiometry (DXA) and air displacement plethysmography. Despite this, we could compare the values they reported because they expressed the results in kilograms (Kg). None of these studies reported changes in LBM.

The studies we used to analyse this outcome had low heterogeneity $\left(\mathrm{Chi}^{2} 1.74, p=0.63 ; I^{2} 0 \%\right.$; $\mathrm{Tau}^{2}=0.00$ ); our meta-analysis found no evidence of a difference in the effect between exercise and control groups (MD $1.01 \mathrm{Kg}[95 \% \mathrm{Cl}-1.78$ to $3.80 \mathrm{Kg}], \mathrm{p}=0.48$, Figure 3). There was a small effect size (SMD 0.09 [-0.27 to 0.44$], p=0.64, I^{2}=0 \%$ ).

\section{DISCUSSION}

Following our results, we established the effect of exercise training in two of the three criteria for sarcopenia in NAFLD patients. Exercise training has a positive impact on physical capacity and no effect on the LBM of NAFLD patients. It was not possible to determine the impact of exercise on NAFLD patients' muscle strength.

\section{Effect of exercise on muscle strength}

Some antecedents show the relationship between NAFLD status and muscle strength. ${ }^{63} \mathrm{~A}$ recent study suggests that a higher hepatic steatosis index (HIS), a clinical feature of NAFLD patients, is associated with lower muscle strength in both sexes. ${ }^{63}$ This antecedent suggests the relevance of muscle strength on NAFLD. Therefore, it is crucial to establish and analyse the influence of factors that could improve muscle strength in patients with NAFLD. There is consistent evidence that resistance training increases muscle strength. ${ }^{64-66}$ Despite clear antecedents that link increased muscle strength with resistance exercise, none of the RCTs included here featured resistance training as a sole intervention. Three studies used a combined exercise protocol (endurance and resistance). ${ }^{59-61}$ Despite the combined training, we expected 
them to evaluate physical qualities such as aerobic capacity and muscular strength; however, all the articles only assessed aerobic capacity, and none of them evaluated muscle strength. For this reason, we could not analyse muscle strength as a criterion of sarcopenia.

Beside the conceptual importance of measuring muscle strength in studies that include resistance training, there are also methodological reasons. In general, muscle strength assessments are more straightforward and require less equipment than direct $\mathrm{VO}_{2 \max }$ or $\mathrm{VO}_{2 \text { peak }}$ determination. Concerning the methodology to measure muscle strength, the handgrip strength (HGS) test is one of the most used evidence-based methods for evaluating muscle strength ${ }^{2}$. HGS is a better predictor of adverse clinical outcomes than muscle mass. ${ }^{67}$ If HGS cannot be used, several alternative methodologies are available: knee flexion/extension, dynamometer, one maximum repetition (1RM), ten maximum repetitions (10RM), Isokinetic evaluation and peak expiratory flow (specific to respiration). ${ }^{2}$

One recent study evaluated the relationship between NAFLD status and HGS using a cohort declared as representative of the general Korean population ${ }^{6368}$. The results indicate that a higher HIS is associated with lower HGS in both sexes. ${ }^{68}$ Therefore, HGS, which is an assessment of general strength in sarcopenia and NAFLD, is quick and easy to perform and should be used as a base procedure for evaluating muscle strength in patients with liver pathologies. ${ }^{268}$

Future research should include muscle strength assessments to investigate the effects of exercise, especially resistance training, in the sarcopenia of NAFLD patients.

\section{Exercise on physical performance}

Physical performance, one of the criteria for a sarcopenia diagnosis, is widely evaluated using the CPET, which provides a global assessment of integrative physiological responses. ${ }^{69} 70$ Results from the metaanalysis in our study indicate that most of the articles showed increased physical performance after the training. Only one study found no changes in $\mathrm{VO}_{2 \text { peak }}$ after exercise, which may be explained by the small sample size. ${ }^{56}$

The overall analysis revealed low heterogeneity and an improvement in physical performance under exercise regardless of its features (type, intensity, duration). Three studies performed endurance training without another intervention, ${ }^{56-58}$ while one article performed combined resistance and endurance training. ${ }^{59}$ The endurance training parameters applied in these studies were consistent with the clinical practice guidelines for managing NAFLD from European associations for the study of the liver, diabetes and obesity (EASL, EASD, EASO); they describe a comprehensive lifestyle approach for treating these patients. ${ }^{71}$

All the studies performed CPET and reported the $\mathrm{VO}_{2 \max }$ or $\mathrm{VO}_{2 \text { peak }}$ as indicators of maximal aerobic capacity, which reflects the respiratory and circulatory systems' ability to supply oxygen to skeletal muscles during exercise. ${ }^{72}$ They predicted an increase in oxygen consumption after endurance training, which induces adaptations in the cardiovascular system and increases mitochondrial biogenesis and capillary density in the skeletal muscles, improving the transport and use of oxygen to generate energy. ${ }^{6473}$ Zenith et al. in 2014 observed increases in $\mathrm{VO}_{2 \text { peak }}$ in cirrhotic patients after eight weeks of endurance training. ${ }^{53}$ This antecedent is consistent with the fact that the primary adaptation to endurance training is cardiorespiratory fitness improvement. ${ }^{74}$ Therefore, our meta-analysis showed that planned and supervised physical exercise improved physical performance in patients with sarcopenia associated with NAFLD. It is essential to mention that these patients should perform regular exercise to preserve its beneficial effects. This recommendation is based on the substantial benefits exercise has on hepatic metabolism and the fat loss induced by endurance training. ${ }^{75-77}$ Thus, training is a therapeutic strategy for improving cardiorespiratory fitness in fatty liver disease. ${ }^{78}$ Furthermore, regular physical activity before liver transplantation is essential to combatting patients' immediate stress post-transplant; it is also a critical determinant in long-term health after hepatic transplant. ${ }^{79}$ 
CPET is the gold standard for assessing cardiorespiratory fitness and functional capacity. Unlike tests that estimate oxygen consumption, CPET measures respiratory, cardiovascular and neuromuscular system function. ${ }^{69} 70$ We excluded one study incorporated in our meta-analysis (in the muscle mass outcome) from the physical performance results, as oxygen consumption was determined from a $2 \mathrm{Km}$ walking test. ${ }^{62}$ In addition to the CPET assessment, the studies on patients with sarcopenia associated with NAFLD could include other validated muscle function tests for primary sarcopenia. For example, the short physical performance battery test (SPPB) evaluates gait, strength and balance through a performance scoring system - it is an easy and quick test to implement. ${ }^{20}$ SPPB can assess the severity of sarcopenia as well as predicts mortality on the liver transplant waitlist. ${ }^{81} 82$ Future studies should use the SPPB to gain additional information on muscle function in NAFLD patients, as the European Association for the Study of the Liver (EASL) suggests. ${ }^{83}$

\section{Effect of exercise on muscle mass}

As shown in Figure 3, exercise does not affect NAFLD patients' LBM - a conclusion based on our analysis of four studies. ${ }^{560-62}$ These studies determined LBM using plethysmography and DXA. Both methods are widely used to assess body composition in research. The studies showed considerable variability, but the variability across the studies was very consistent. The lack of significance in the overall effect may be because DXA determinations have low reproducibility, dependent upon the equipment used. The gold standard methods suggested for measuring body composition, especially for muscular mass, are CT and magnetic resonance imaging (MRI). ${ }^{18}$

Considering that all the studies in the meta-analysis mainly comprised endurance training protocols as the central exercise intervention, it is possible that endurance training does not affect NAFLD patients' LBM. Our results are consistent with the findings of a systematic review in cirrhotic patients trained with endurance protocols, which reported no changes in LBM. ${ }^{94}$ Contrary to these results, a study in cirrhotic patients featuring resistance training showed an increase in muscle size. ${ }^{85}$ This observation suggests that the type of exercise training determines the effects on muscle mass, and that resistance training could have a positive impact on muscle mass in NAFLD patients.

\section{Exercise conditions to regulate sarcopenia}

Therapies to revert muscular mass loss in NAFLD patients include pharmacological and nutritional approaches. However, exercise-based treatments should not be excluded because they can improve the patients' generalised physical condition and life quality. ${ }^{8}$ It is widely established that resistance training is the most promising method to increase muscle strength and mass as well as balance; ${ }^{86}$ further, it can induce muscular hypertrophy, translating to increased muscle mass. ${ }^{86}$ Based on these antecedents, resistance training should be indicated as a therapy, alone or in combination, for sarcopenia in NAFLD patients. It is necessary to consider the conditions of resistance training to use in NAFLD patients. The standard conditions suggest that a high load induces a higher hypertrophic response in skeletal muscle; ${ }^{87}$ however, loads below $30 \%$ of 1 RPM seem sufficient to trigger a hypertrophic response. The current recommendation for hypertrophic training is an intensity of $40-80 \%$ of the individual $1 \mathrm{RM}$, with loads $>60 \%$ to increase maximal force and muscular mass. ${ }^{88}$ Thus, the best conditions for resistance training in NAFLD patients require further study.

\section{Limitations}

As mentioned, the main limitations of the RCTs included here were difficulty in blinding participants, therapists and assessors and participants' non-adherence to the assigned intervention regimes. Blinding participants and researchers are a significant challenge in exercise RCTs, as lifestyle interventions require patients and therapists to be fully aware of the type of intervention to be delivered. Nonetheless, for reducing the risk of bias is crucial to blind the outcome assessors, which must be clearly stated in the RCT 
report. As for non-adherence, researchers should include an intention to treat analyses in a pre-specified fashion, considering that attrition is a common feature in exercise RCTs.

Another limitation was the lack of supervision for not performing the physical activity in the control group. This monitoring is essential to ensuring a low level of physical activity that does not influence the results. If this variable is not controlled, then the sedentary controls could indeed be individuals who belong to a group that performs an unsupervised and undeclared exercise. These limitations should be considered in future RCTs.

The lack of categorisation of NAFLD severity in the RCT participants is another major limitation of the studies included here. This categorisation is fundamental to determine if the effect of exercise training on sarcopenia depends on the stage of the disease. Future RCTs should include this classification when planning and executing exercise protocols.

The exercise training programs were significantly different among the studies regarding exercise intensity, total duration, session frequency and modality (endurance or combined training). Among these, training modality had special consideration, since resistance training is known to be one of the most potent interventions for increasing muscle size and quality in healthy and ill subjects. ${ }^{89}$ This fact is caused by increased synthesis of myofibrillar and mitochondrial proteins in untrained individuals and by an increase in strength, muscle mass and effort tolerance. ${ }^{64-667490}$ Nevertheless, none of the studies used resistance training to treat NAFLD patients. Therefore, we strongly recommend including resistance training as the main component of exercise protocols in future research on sarcopenia in NAFLD patients.

\section{CONCLUSION}

Exercise training is a useful tool to treat sarcopenia in NAFLD patients; it increases physical performance in the form of aerobic capacity. Nevertheless, it does not affect LBM. Future research should include muscle strength assessments and resistance training to evaluate the effects of exercise training on sarcopenia in NAFLD patients.

\section{WHAT IS ALREADY KNOWN?}

- Sarcopenia negatively affects NAFLD progression.

- Previous RCTs and meta-analyses have reported beneficial effects from exercise on liver dysfunction in NAFLD patients.

\section{WHAT ARE THE NEW FINDINGS?}

- There are statistically significant effects of exercise interventions on sarcopenia in NAFLD patients.

- Exercise training has a positive effect on physical performance (aerobic capacity) but does not affect NAFLD patients' LBM. After the initial screening of the RCTs, we cannot include muscle strength as an outcome because none of these RCTs evaluate it.

\section{Contributors}

All authors comply with the ICMJE Recommendations of authorship. A.G., C.C-V., F.S., and O.A. had full access to all of the data in the study and takes responsibility for the integrity of the data and the accuracy of the data analysis, O.A. contributed with the study design, to statistical analysis and interpretation of the results, and C.H-S., F.S. and M.V-B. contributed to the study design and writing of the manuscript. All authors approved the final version to be published.

\section{Competing interests}

None declared. 


\section{Funding}

The manuscript was supported by research grants from the National Fund for Science and Technological Development (FONDECYT 1200944 [CC-V], 1201039 [FS]), the Millennium Institute on Immunology and Immunotherapy (P09-016-F [CC-V, FS]) and BASAL Grant - CEDENNA from the National Research and Development Agency (ANID), Government of Chile (AFB180001 [CC-V]). The Millennium Nucleus of Ion Channels-Associated Diseases (MiNICAD) is supported by the Iniciativa Científica Milenio (ANID, Chile).

\section{Acknowledgment}

We acknowledge Orlando Flores Guerrero PhD., Faculty of Health and Behavioural Sciences, The University of Queensland, for his input while drafting the manuscript.

\section{FIGURE LEGENDS}

Figure 1. Flow chart of search results and study selection according to the PRISMA statement.

Figure 2. A meta-analysis of pooled effect size and confidence intervals ( $\mathrm{Cls}$ ) (95\%) of the interventions with exercise versus control group on physical performance evaluated by the cardiopulmonary exercise test (CPET) $(\mathrm{mL} / \mathrm{Kg} * \mathrm{~min})$.

Figure 3. A meta-analysis of pooled effect size (ES) and confidence intervals ( $\mathrm{Cls})(95 \%)$ of the intervention with exercise versus control group on lean mass evaluated by dual-energy X-ray absorptiometry (DXA) and air displacement plethysmograph (Kg).

Supplementary Material 1. Search strategy in PubMed, CINAHL and Scopus databases.

Supplementary Material 2. Details of the studies included in the systematic review and meta-analysis.

Supplementary Material 3. Causes for exclusion for each excluded randomised controlled trial.

Supplementary Material 4. Methodological quality of the included studies in physical performance analysis. Methodological quality of the randomised controlled trials $(n=4)$ was assessed using the Cochrane risk of bias tool (six evaluation-critical methodological components).

Supplementary Material 5. Methodological quality of the included studies in lean body mass (LBM) analysis. Methodological quality of the randomised controlled trials $(n=4)$ was assessed using the Cochrane risk of bias tool (six evaluation-critical methodological components). 


\section{REFERENCES}

1. Chalasani Naga YZ, Lavine Joel E., Mae Diehl Anna, Brunt Elizabeth M., Cusi Kenneth, Charlton Michael and Sanyal Arun J. The diagnosis and management of non-alcoholic fatty liver disease: practice Guideline by the American Association for the Study of Liver Diseases, American College of Gastroenterology, and the American Gastroenterological Association. Hepatology 2012;55(6):2005-23. doi: 10.1002/hep.25762

2. El Sherif O, Dhaliwal A, Newsome PN, et al. Sarcopenia in nonalcoholic fatty liver disease: new challenges for clinical practice. Expert Rev Gastroenterol Hepatol 2020;14(3):197-205. doi: 10.1080/17474124.2020.1731303

3. Yu R, Shi Q, Liu L, et al. Relationship of sarcopenia with steatohepatitis and advanced liver fibrosis in non-alcoholic fatty liver disease: a meta-analysis. BMC Gastroenterol 2018;18(1):51. doi: 10.1186/s12876-018-0776-0

4. Cruz-Jentoft AJ, Bahat G, Bauer J, et al. Sarcopenia: revised European consensus on definition and diagnosis. Age Ageing 2019;48(4):601. doi: 10.1093/ageing/afz046 [published Online First: 2019/05/14]

5. Lee YH, Jung KS, Kim SU, et al. Sarcopaenia is associated with NAFLD independently of obesity and insulin resistance: Nationwide surveys (KNHANES 2008-2011). I Hepatol 2015;63(2)(1600-0641 (Electronic)):486-93. doi: 10.1016/j.jhep.2015.02.051

6. Dasarathy S. Treatment to Improve Nutrition and Functional Capacity Evaluation in Liver Transplant Candidates. Curr Treat Options Gastroenterol 2014;12(2):242-55. doi: 10.1007/s11938-014-00169

7. Meeks AC, Madill J. Sarcopenia in liver transplantation: A review. Clinical Nutrition ESPEN 2017;22:7680. doi: 10.1016/j.clnesp.2017.08.005

8. Tandon P, Dunn MA, Duarte-Rojo A. Resistance Training Reduces Risk of Sarcopenia in Patients With Cirrhosis. Clin Gastroenterol Hepatol 2020;18(5)(1542-7714 (Electronic)):1036-39. doi: 10.1016/j.cgh.2019.09.030

9. Aamann L, Dam G, Rinnov AR, et al. Physical exercise for people with cirrhosis. Cochrane Database Syst Rev 2018;2018(12) doi: 10.1002/14651858.CD012678.pub2

10. Smart NA, King N, McFarlane JR, et al. Effect of exercise training on liver function in adults who are overweight or exhibit fatty liver disease: a systematic review and meta-analysis. Br J Sports Med 2018;52(13):834-43. doi: 10.1136/bjsports-2016-096197

11. Medrano M, Cadenas-Sanchez C, Álvarez-Bueno C, et al. Evidence-Based Exercise Recommendations to Reduce Hepatic Fat Content in Youth- a Systematic Review and Meta-Analysis. Prog CardiovasC Dis 2018;61(2)(1873-1740 (Electronic)):222-31. doi: 10.1016/j.pcad.2018.01.013.

12. Guyatt GH, Oxman AD, Kunz R, et al. GRADE guidelines: 2. Framing the question and deciding on important outcomes. Journal of Clinical Epidemiology 2011;64(4):395-400. doi: 10.1016/j.jclinepi.2010.09.012

13. Younossi ZM. Nonalcoholic fatty liver disease and nonalcoholic steatohepatitis: Implications for liver transplantation. Liver Transplantation: Official Publication of the American Association for the Study of Liver Diseases and the International Liver Transplantation Society 2018;24(2):166-70. doi: 10.1002/It.25003

14. Perumpail BJ, Khan MA, Yoo ER, et al. Clinical epidemiology and disease burden of nonalcoholic fatty liver disease. World Journal of Gastroenterology 2017;23(47):8263-76. doi: 10.3748/wjg.v23.i47.8263

15. van Deursen VM, Damman K, Hillege $\mathrm{HL}$, et al. Abnormal liver function in relation to hemodynamic profile in heart failure patients. Journal of Cardiac Failure 2010;16(1):84-90. doi: 10.1016/j.cardfail.2009.08.002 
16. Kong L-Z, Chandimali N, Han Y-H, et al. Pathogenesis, Early Diagnosis, and Therapeutic Management of Alcoholic Liver Disease. International Journal of Molecular Sciences 2019;20(11) doi: 10.3390/ijms20112712

17. Engelking LR, Dasher CA, Hirschowitz BI. Within-day Fluctuations in Serum Bile-acid Concentrations among Normal Control Subjects and Patients with Hepatic Disease. American Journal of Clinical Pathology 1980;73(2):196-201. doi: 10.1093/ajcp/73.2.196

18. Cruz-Jentoft AJ, Bahat G, Bauer J, et al. Sarcopenia: revised European consensus on definition and diagnosis. Age Ageing 2019;48(1):16-31. doi: 10.1093/ageing/afy169

19. Suchomel TJ, Nimphius S, Stone MH. The Importance of Muscular Strength in Athletic Performance. Sports Med 2016;46(10):1419-49. doi: 10.1007/s40279-016-0486-0 [published Online First: 2016/02/04]

20. Heymsfield SB, McManus C, Smith J, et al. Anthropometric measurement of muscle mass: revised equations for calculating bone-free arm muscle area. Am J Clin Nutr 1982;36(4):680-90. doi: 10.1093/ajcn/36.4.680 [published Online First: 1982/10/01]

21. Beaudart C, Rolland Y, Cruz-Jentoft AJ, et al. Assessment of Muscle Function and Physical Performance in Daily Clinical Practice : A position paper endorsed by the European Society for Clinical and Economic Aspects of Osteoporosis, Osteoarthritis and Musculoskeletal Diseases (ESCEO). Calcif Tissue Int 2019;105(1):1-14. doi: 10.1007/s00223-019-00545-w [published Online First: 2019/04/12]

22. Smart NA, Waldron M Fau - Ismail H, Ismail H Fau - Giallauria F, et al. Validation of a new tool for the assessment of study quality and reporting in exercise training studies: TESTEX. Int J Evid Based Healthc 2015;13(1)(1744-1609 (Electronic)):9-18. doi: 10.1097/XEB.0000000000000020.

23. Oxfeldt $M$, Overgaard $K$, Hvid $L$, et al. Effects of plyometric training on jumping, sprint performance and lower body muscle strength in healthy adults: A systematic review and meta-analyses. Scandinavian Journal of Medicine and Science in Sports 2019;29 doi: 10.1111/sms.13487

24. Review Manager (RevMan) [Computer program] [program]. 5.4 version: The Cochrane Collaboration, 2020.

25. Takeshima N, Sozu T, Tajika A, et al. Which is more generalizable, powerful and interpretable in metaanalyses, mean difference or standardized mean difference? BMC Medical Research Methodology 2014;14(1):30. doi: 10.1186/1471-2288-14-30

26. Cohen J. Statistical Power Analysis for the Behavioral Sciences. New York: Routledge 1988.

27. Higgins JP, Thompson SG. Quantifying heterogeneity in a meta-analysis. Stat Med 2002;21(11)(02776715 (Print)):1539-58. doi: 10.1002/sim.1186.

28. Franco I, Bianco A, Diaz MdP, et al. Eficiencia de dos programas de actividad fisica sobre la esteatosis hepatica no alcoholica. un trial clinico randomizato. Revista de la Facultad de Ciencias Médicas de Córdoba 2019;76(1):26-36. doi: 10.31053/1853.0605.v76.n1.21638

29. Katsagoni CN, Papatheodoridis GV, loannidou P, et al. Improvements in clinical characteristics of patients with non-alcoholic fatty liver disease, after an intervention based on the Mediterranean lifestyle: a randomised controlled clinical trial. Br J Nutr 2018;164-175(1475-2662 (Electronic)) doi: 10.1017/S000711451800137X

30. Axley P, Kodali S, Kuo Y-F, et al. Text Messaging Approach Improves Weight Loss in Patients with Nonalcoholic Fatty Liver Disease: A Randomized Study. Liver International 2017;38 doi: 10.1111/liv.13622

31. Draz RS, Serry ZMH, Rahmy AF, et al. Electroacupuncture Versus Aerobic Interval Training on Liver Functions in Patients with Nonalcoholic Fatty Liver. J Altern Complement Med 2020;26(1)(15577708 (Electronic)) doi: https://doi.org/10.1089/acm.2019.0182

32. Abd El-Kader SM, Al-Shreef FM, Al-Jiffri OH. Biochemical parameters response to weight loss in patients with non-alcoholic steatohepatitis. Afr Health Sci 2016;16(1):242-49. doi: 10.4314/ahs.v16i1.32 
33. Skrypnik D, Ratajczak M, Karolkiewicz J, et al. Effects of endurance and endurance-strength exercise on biochemical parameters of liver function in women with abdominal obesity. Biomed Pharmacother 2016;80(1950-6007 (Electronic)):1-7. doi: 10.1016/j.biopha.2016.02.017

34. Yoshimura E, Kumahara H, Tobina $\mathrm{T}$, et al. Lifestyle Intervention Involving Calorie Restriction with or without Aerobic Exercise Training Improves Liver Fat in Adults with Visceral Adiposity. Journal of Obesity 2014;2014:197216. doi: 10.1155/2014/197216

35. Zelber-Sagi S, Buch A, Yeshua $\mathrm{H}$, et al. Effect of resistance training on non-alcoholic fatty-liver disease a randomized-clinical trial. World J Gastroenterol 2014;20(15)(2219-2840 (Electronic)):4382-92. doi: 10.3748/wjg.v20.i15.4382

36. Straznicky N, Lambert E, Grima M, et al. The effects of dietary weight loss with or without exercise training on liver enzymes in obese metabolic syndrome subjects. Diabetes, obesity \& metabolism 2011;14:139-48. doi: 10.1111/j.1463-1326.2011.01497.x

37. George A, Bauman A, Johnston A, et al. Effect of a lifestyle intervention in patients with abnormal enzymes and metabolic risk factors. Journal of gastroenterology and hepatology 2009;24:399-407. doi: 10.1111/j.1440-1746.2008.05694.x

38. Promrat K, Kleiner De Fau - Niemeier HM, Niemeier Hm Fau - Jackvony E, et al. Randomized controlled trial testing the effects of weight loss on nonalcoholic steatohepatitis. Hepatology 2010;51(1)(1527-3350 (Electronic)):121-9. doi: 10.1002/hep.23276

39. Winn NC, Liu Y, Rector RS, et al. Energy-matched moderate and high intensity exercise training improves nonalcoholic fatty liver disease risk independent of changes in body mass or abdominal adiposity - A randomized trial. Metabolism 2018;78:128-40. doi: https://doi.org/10.1016/i.metabol.2017.08.012

40. Garcia AMC, Veneroso CE, Soares DD, et al. Effect of a Physical Exercise Program on the Functional Capacity of Liver Transplant Patients. Transplantation Proceedings 2014;46(6):1807-08. doi: https://doi.org/10.1016/j.transproceed.2014.05.023

41. Rachakonda V, Wills R, DeLany JP, et al. Differential Impact of Weight Loss on Nonalcoholic Fatty Liver Resolution in a North American Cohort with Obesity. Obesity (Silver Spring) 2017;25(8):1360-68. doi: 10.1002/oby. 21890

42. Galbreath M, Campbell, B., LaBounty, P., Bunn, J., Dove, J., Harvey, T., Hudson, G., Gutierrez, J., Levers, K., Galvan, E., Jagim, A., Greenwood, L., Cooke, M., Greenwood, M., Rasmussen, C., \& Kreider, R. Effects of Adherence to a Higher Protein Diet on Weight Loss, Markers of Health, and Functional Capacity in Older Women Participating in a Resistance-Based Exercise Program. Nutrients 2018;10(8) doi: http://dx.doi.org/10.3390/nu10081070

43. Piano A, De Mello M, Sanches $P$, et al. Long-term effects of aerobic plus resistance training on the adipokines and neuropeptides in nonalcoholic fatty liver disease obese adolescents. European journal of gastroenterology \& hepatology 2012;24:1313-24. doi: 10.1097/MEG.0b013e32835793ac

44. Lee S, Bacha F, Hannon T, et al. Effects of Aerobic Versus Resistance Exercise Without Caloric Restriction on Abdominal Fat, Intrahepatic Lipid, and Insulin Sensitivity in Obese Adolescent Boys. Diabetes 2012;61(11):2787. doi: 10.2337/db12-0214

45. Sánchez-Muñoz V, Salas-Romero R Fau - Del Villar-Morales A, Del Villar-Morales A Fau - Martínez-Coria $E$, et al. [Decrease of liver fat content by aerobic exercise or metformin therapy in overweight or obese women]. Rev Invest Clin 2013;65(4)(0034-8376 (Print)):307-17.

46. Brouwers BA-O, Schrauwen-Hinderling VB, Jelenik T, et al. Exercise training reduces intrahepatic lipid content in people with and people without nonalcoholic fatty liver. Am J Physiol Endocrinol Metab 2018;314(2)(1522-1555 (Electronic)):E165-E73. doi: 10.1152/ajpendo.00266.2017 
47. Pugh CJA, Sprung VS, Jones $\mathrm{H}$, et al. Exercise-induced improvements in liver fat and endothelial function are not sustained 12 months following cessation of exercise supervision in nonalcoholic fatty liver disease. International Journal of Obesity 2016;40(12):1927-30. doi: 10.1038/ijo.2016.123

48. Debette-Gratien M, Tabouret T, Antonini M-T, et al. Personalized Adapted Physical Activity Before Liver Transplantation: Acceptability and Results. Transplantation 2015;99(1)

49. Devries MC, Samjoo la Fau - Hamadeh MJ, Hamadeh Mj Fau - Tarnopolsky MA, et al. Effect of endurance exercise on hepatic lipid content, enzymes, and adiposity in men and women. Obesity (Silver Spring) 2008;16(10)(1930-7381 (Print)):2281-8. doi: 10.1038/oby.2008.358

50. Zhang H-J, Pan L-L, Ma Z-M, et al. Long-term effect of exercise on improving fatty liver and cardiovascular risk factors in obese adults: A 1-year follow-up study. Diabetes, Obesity and Metabolism 2017;19(2):284-89. doi: 10.1111/dom.12809

51. Abdelbasset WK, Tantawy SA, Kamel DM, et al. Effects of high-intensity interval and moderate-intensity continuous aerobic exercise on diabetic obese patients with nonalcoholic fatty liver disease: A comparative randomized controlled trial. Medicine 2020;99(10)

52. Kim H, Kim M, Kojima N, et al. Exercise and Nutritional Supplementation on Community-Dwelling Elderly Japanese Women With Sarcopenic Obesity: A Randomized Controlled Trial. J Am Med Dir Assoc 2016;17(11)(1538-9375 (Electronic)):1011-19. doi: 10.1016/j.jamda.2016.06.016

53. Zenith L, Meena N, Ramadi A, et al. Eight weeks of exercise training increases aerobic capacity and muscle mass and reduces fatigue in patients with cirrhosis. Clin Gastroenterol Hepatol 2014;12(11):1920-26.e2. doi: 10.1016/j.cgh.2014.04.016

54. Johnson NA, Sachinwalla T, Walton DW, et al. Aerobic exercise training reduces hepatic and visceral lipids in obese individuals without weight loss. Hepatology 2009;50(4):1105-12. doi: 10.1002/hep. 23129

55. Yoo HJ, Hwang SY, Cho GJ, et al. Association of Glypican-4 With Body Fat Distribution, Insulin Resistance, and Nonalcoholic Fatty Liver Disease. The Journal of Clinical Endocrinology \& Metabolism 2013;98(7):2897-901. doi: 10.1210/jc.2012-4297

56. Sullivan S, Kirk Ep Fau - Mittendorfer B, Mittendorfer B Fau - Patterson BW, et al. Randomized trial of exercise effect on intrahepatic triglyceride content and lipid kinetics in nonalcoholic fatty liver disease. Hepatology 2012;55(6)(1527-3350 (Electronic)):1738-45. doi: doi: 10.1002/hep.25548

57. Pugh CJA, Cuthbertson DJ, Sprung VS, et al. Exercise training improves cutaneous microvascular function in nonalcoholic fatty liver disease. American journal of physiology Endocrinology and metabolism 2013;305:E50-E58. doi: 10.1152/ajpendo.00055.2013.

58. Pugh CJA, Sprung VS, Kemp GJ, et al. Exercise training reverses endothelial dysfunction in nonalcoholic fatty liver disease. Am J Physiol Heart Circ Physiol 2014;307:H1298-H306. doi: 10.1152/ajpheart.00306.2014.

59. Shojaee-Moradie F, Cuthbertson DJ, Barrett M, et al. Exercise Training Reduces Liver Fat and Increases Rates of VLDL Clearance But Not VLDL Production in NAFLD. The Journal of Clinical Endocrinology \& Metabolism 2016;101(11):4219-28. doi: 10.1210/jc.2016-2353

60. Hallsworth K, Thoma C, Hollingsworth KG, et al. Modified high-intensity interval training reduces liver fat and improves cardiac function in non-alcoholic fatty liver disease: a randomized controlled trial. Clin Sci (Lond) 2015;129(12)(1470-8736 (Electronic)):1097-105. doi: 10.1042/CS20150308

61. Houghton D, Thoma C, Hallsworth K, et al. Exercise Reduces Liver Lipids and Visceral Adiposity in Patients With Nonalcoholic Steatohepatitis in a Randomized Controlled Trial. Clin Gastroenterol Hepatol 2017;15(1)(1542-7714 (Electronic)):96-102.e3. doi: 10.1016/j.cgh.2016.07.031

62. Cheng SA-O, Ge J, Zhao C, et al. Effect of aerobic exercise and diet on liver fat in pre-diabetic patients with non-alcoholic-fatty-liver-disease: A randomized controlled trial. Sci Rep 2017;7(1)(2045-2322 (Electronic)) doi: 10.1038/s41598-017-16159-x. 
63. Kim JA, Choi KM. Sarcopenia and fatty liver disease. Hepatol Int 2019;13(6):674-87. doi: 10.1007/s12072-019-09996-7

64. Hawley JA, Hargreaves M, Joyner MJ, et al. Integrative biology of exercise. Cell 2014;159(4):738-49. doi: 10.1016/j.cell.2014.10.029

65. Liu TC-Y, Liu G, Hu S-J, et al. Quantitative Biology of Exercise-Induced Signal Transduction Pathways. Adv Exp Med Biol 2017;977:419-24. doi: 10.1007/978-3-319-55231-6_54

66. Francaux M, Deldicque L. Exercise and the control of muscle mass in human. Pflugers Arch 2019;471(3):397-411. doi: 10.1007/s00424-018-2217-x

67. Lauretani F, Russo Cr Fau - Bandinelli S, Bandinelli S Fau - Bartali B, et al. Age-associated changes in skeletal muscles and their effect on mobility: an operational diagnosis of sarcopenia. J Appl Physiol (1985) 2003;95(5)(8750-7587 (Print)):1851-60. doi: 10.1152/japplphysiol.00246.2003.

68. Kim BJ, Ahn SH, Lee SH, et al. Lower hand grip strength in older adults with non-alcoholic fatty liver disease: a nationwide population-based study. Aging (Albany NY) 2019;11(13)(1945-4589 (Electronic)):4547-60. doi: 10.18632/aging.102068

69. Milani RV, Lavie CJ, Mehra MR, et al. Understanding the Basics of Cardiopulmonary Exercise Testing. Mayo Clinic Proceedings 2006;81(12):1603-11. doi: 10.4065/81.12.1603

70. Weisman IM, Weisman IM, Marciniuk D, et al. ATS/ACCP Statement on Cardiopulmonary Exercise Testing. American Journal of Respiratory and Critical Care Medicine 2003;167(2):211-77. doi: 10.1164/rccm.167.2.211

71. Marchesini G, Day CP, Dufour JF, et al. EASL-EASD-EASO Clinical Practice Guidelines for the Management of Non-Alcoholic Fatty Liver Disease. Obesity Facts 2016;9(2)(1662-4033 (Electronic)):56-90. doi: https://doi.org/10.1159/000443344

72. Lee I, Kim J, Kang HA-O. Estimated Cardiorespiratory Fitness Attenuates the Impacts of Sarcopenia and Obesity on Non-Alcoholic Fatty Liver in Korean Adults. LID - 10.3390/ijerph17113902 [doi] LID 3902. Int J Environ Res Public Health 2020;17(11)(1660-4601 (Electronic)) doi: https://doi.org/10.3390/ijerph17113902

73. Hughes DC, Ellefsen S, Baar K. Adaptations to Endurance and Strength Training. LID 10.1101/cshperspect.a029769 [doi] LID - a029769. Cold Spring Harb Perspect Med 2018;8(6)(2157-1422 (Electronic)) doi: 10.1101/cshperspect.a029769.

74. McGee SL, Hargreaves M. Exercise adaptations: molecular mechanisms and potential targets for therapeutic benefit. Nature Reviews Endocrinology 2020 doi: 10.1038/s41574-020-0377-1

75. Lo MS, Lin LI Fau - Yao W-J, Yao Wj Fau - Ma M-C, et al. Training and detraining effects of the resistance vs. endurance program on body composition, body size, and physical performance in young men. $J$ Strength Cond Res 2011;25(8)(1533-4287 (Electronic)):2246-54. doi: 10.1519/JSC.0b013e3181e8a4be.

76. Nolan PA-O, Keeling SM, Robitaille CA, et al. The Effect of Detraining after a Period of Training on Cardiometabolic Health in Previously Sedentary Individuals. LID - 10.3390/ijerph15102303 [doi] LID - 2303. International Journal of Environmental Research and Public Health 2018;15(10)(16604601 (Electronic)) doi: https://doi.org/10.3390/ijerph15102303

77. Brouwers $B$, Hesselink $M K$, Schrauwen $P$, et al. Effects of exercise training on intrahepatic lipid content in humans. Diabetologia 2016;59(10)(1432-0428 (Electronic)):2068-79. doi: 10.1007/s00125-0164037-x.

78. van der Windt DJ, Sud V, Zhang H, et al. The Effects of Physical Exercise on Fatty Liver Disease. Gene Expr 2018;18(2):89-101. doi: 10.3727/105221617X15124844266408

79. Dunn MA-O, Rogal SA-O, Duarte-Rojo AA-O, et al. Physical Function, Physical Activity, and Quality of Life After Liver Transplantation. Liver Transp/ 2020;26(5)(1527-6473 (Electronic)):702-08. doi: 10.1002/lt.25742 
medRxiv preprint doi: https://doi.org/10.1101/2020.09.05.20189100; this version posted September 11 , 2020. The copyright holder for this preprint (which was not certified by peer review) is the author/funder, who has granted medRxiv a license to display the preprint in perpetuity.

It is made available under a CC-BY-NC-ND 4.0 International license .

80. Phu S, Kirk B, Bani Hassan E, et al. The diagnostic value of the Short Physical Performance Battery for sarcopenia. BMC Geriatrics 2020;20(1):242. doi: 10.1186/s12877-020-01642-4

81. Lai JC, Feng S, Terrault NA, et al. Frailty predicts waitlist mortality in liver transplant candidates. American journal of transplantation : official journal of the American Society of Transplantation and the American Society of Transplant Surgeons 2014;14(8):1870-9. doi: 10.1111/ajt.12762 [published Online First: 2014/06/18]

82. Wang CW, Feng S Fau - Covinsky KE, Covinsky Ke Fau - Hayssen H, et al. A Comparison of Muscle Function, Mass, and Quality in Liver Transplant Candidates: Results From the Functional Assessment in Liver Transplantation Study. Transplantation 2016;100(8)(1534-6080 (Electronic)):1692-8. doi: 10.1097/TP.0000000000001232

83. European Association for the Study of the Liver. Electronic address eee, European Association for the Study of the L. EASL Clinical Practice Guidelines on nutrition in chronic liver disease. J Hepatol 2019;70(1):172-93. doi: 10.1016/j.jhep.2018.06.024 [published Online First: 2018/08/27]

84. Naseer $M$, Turse EP, Syed A, et al. Interventions to improve sarcopenia in cirrhosis: A systematic review. World J Clin Cases 2019;7(2):156-70. doi: 10.12998/wjcc.v7.i2.156

85. Aamann L, Dam G, Borre M, et al. Resistance Training Increases Muscle Strength and Muscle Size in Patients With Liver Cirrhosis. Clin Gastroenterol Hepatol 2019;18(5)(1542-7714 (Electronic)):117987.e6. doi: 10.1016/j.cgh.2019.07.058.

86. Giallauria F, Cittadini A Fau - Smart NA, Smart Na Fau - Vigorito C, et al. Resistance training and sarcopenia. Monaldi Arch Chest Dis 2016;84(1-2)(1122-0643 (Print)) doi: 10.4081/monaldi.2015.738

87. Csapo R, Alegre LA-O. Effects of resistance training with moderate vs heavy loads on muscle mass and strength in the elderly: A meta-analysis. Scand J Med Sci Sports 2016;26(9)(1600-0838 (Electronic)):995-1006. doi: 10.1111/sms.12536

88. Wackerhage $\mathrm{H}$, Schoenfeld BJ, Hamilton DL, et al. Stimuli and sensors that initiate skeletal muscle hypertrophy following resistance exercise. J Appl Physiol (1985) 2019;126(1):30-43. doi: 10.1152/japplphysiol.00685.2018

89. Pescatello L. ACSM's Guidelines for Exercise Testing and Prescription 9th Ed. 2014. J Can Chiropr Assoc 2014;58(3):328.

90. Wilkinson SB, Phillips Sm Fau - Atherton PJ, Atherton Pj Fau - Patel R, et al. Differential effects of resistance and endurance exercise in the fed state on signalling molecule phosphorylation and protein synthesis in human muscle. (1469-7793 (Electronic)) 


\section{든 $\quad$ Records identified through}

database searching $(n=66)$

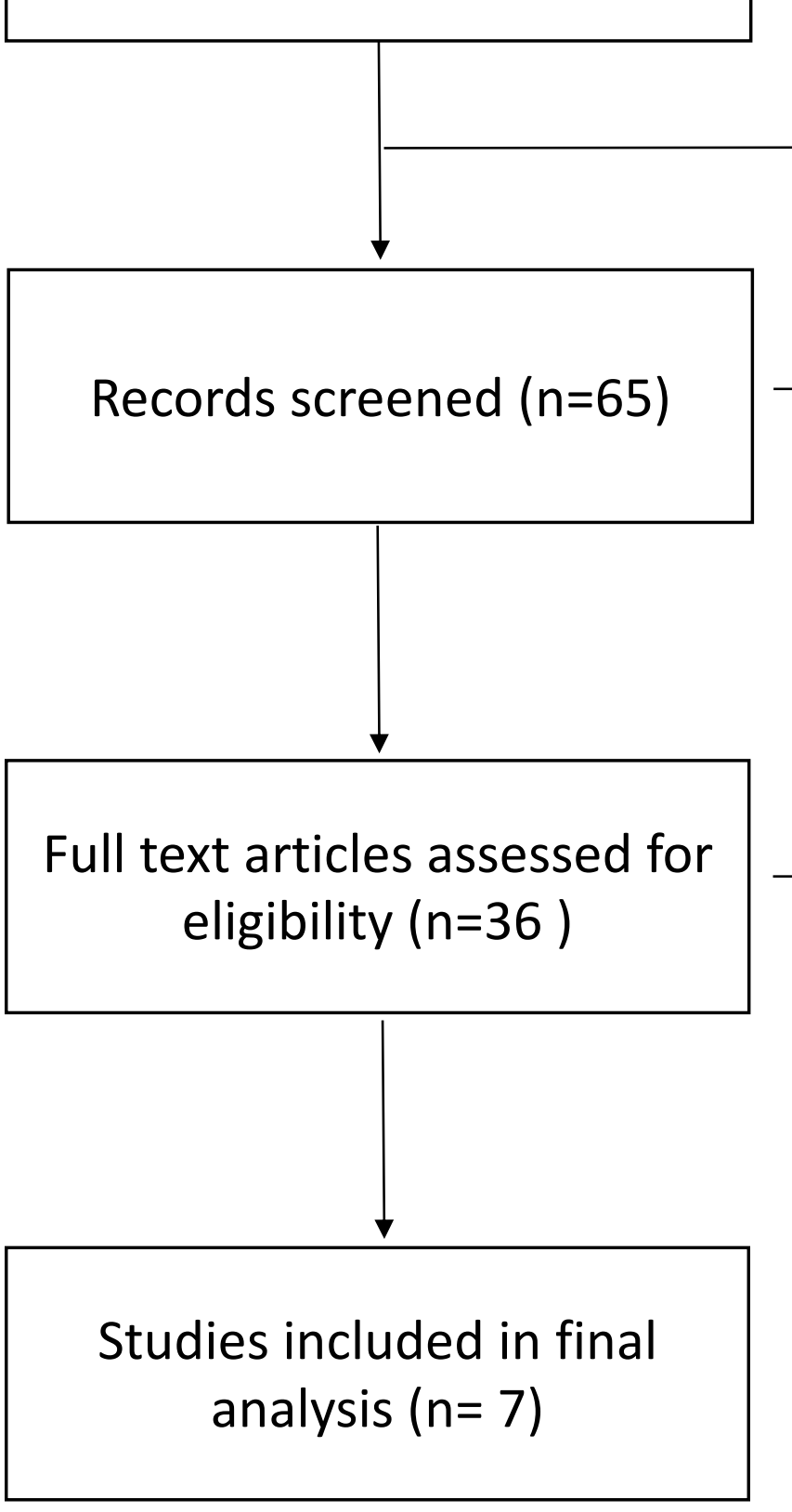

- Pubmed $(n=44)$

- CINAHL $(n=21)$

- Scopus $(n=1)$

\section{Duplicate articles $(n=1)$}

Records excluded based on review of title and/or abstract

$$
(n=29)
$$

Criteria to exclude full text articles $(n=29)$ :

- Exercise combined with another intervention $(n=15)$

- Subjects under 18 years of age $(n=2)$

- Article in Spanish $(n=1)$

- Not RCT $(n=5)$

- Not evaluate at least 1 sarcopenia criteria $(n=2)$

- Lack clearly diagnosed NAFLD or that had comorbidities $(n=4)$ 


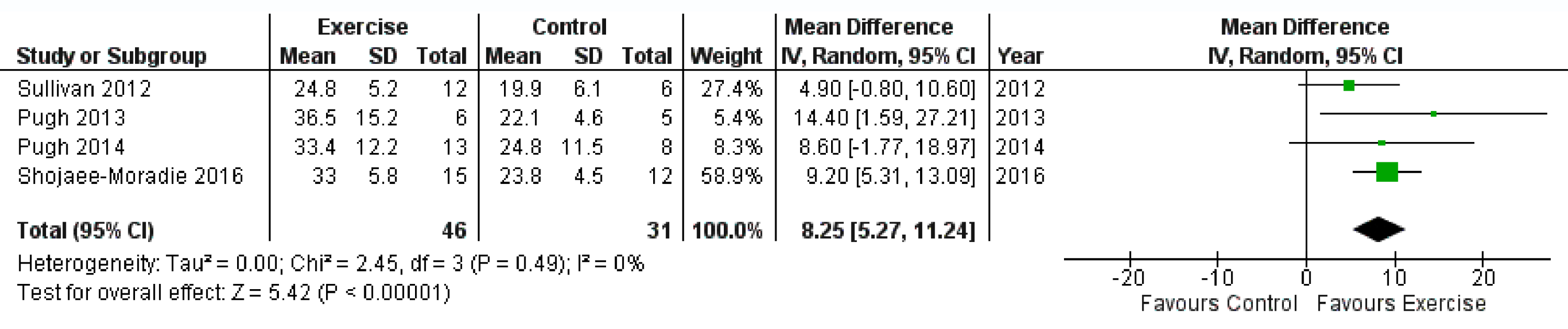

Figure 2 


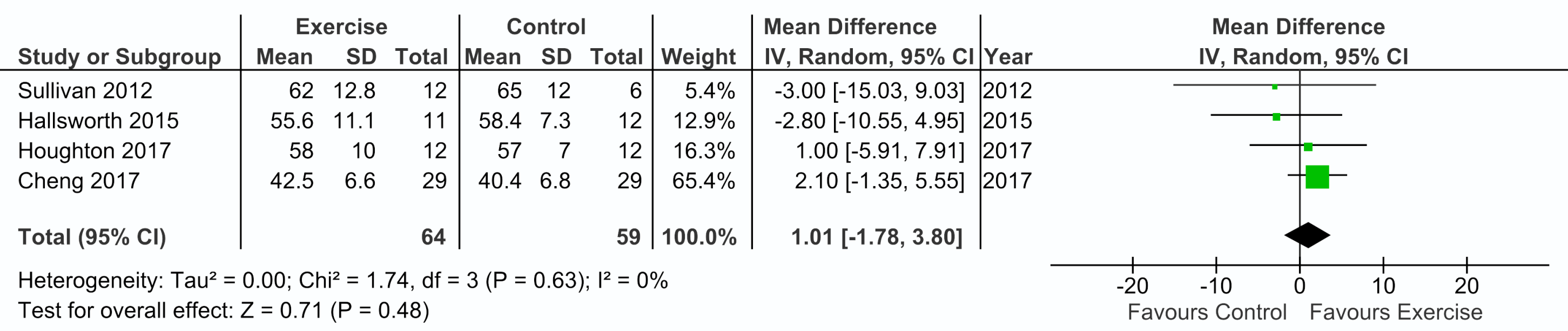

Figure 3 